\title{
TYPE Ia SUPERNOVA PROGENITORS AND CHEMICAL ENRICHMENT IN HYDRODYNAMICAL SIMULATIONS. I. THE SINGLE-DEGENERATE SCENARIO
}

\author{
Noelia Jiménez ${ }^{1,2,3,4}$, Patricia B. Tissera ${ }^{2,5,6}$, and Francesca Matteucci ${ }^{3,7,8}$ \\ ${ }^{1}$ School of Physics \& Astronomy, University of St. Andrews, North Haugh, St. Andrews, KY16 9SS, Scotland, UK; nj22@st-andrews.ac.uk \\ ${ }^{2}$ Instituto de Astronomía y Física del Espacio (IAFE, CONICET-UBA), CC. 67 Suc. 28, C1428ZAA, Ciudad de Buenos Aires, Argentina \\ ${ }^{3}$ Dipartimento di Fisica, Universita' di Trieste, Via G. B. Tiepolo, 11, I-34100, Trieste, Italy \\ ${ }^{4}$ Institut d' Estudis Espacials de Catalunya (IEEC), Institut de Ciencies de L'Espai (ICE), Spain \\ ${ }^{5}$ Departamento de Ciencias Fisicas, Universidad Andres Bello, Av. Republica 220, Santiago, Chile \\ ${ }^{6}$ Millenium Institute of Astrophysics, Santiago, Chile \\ ${ }^{7}$ INAF, Trieste, Via G. B. Tiepolo, 11, I-34100, Trieste, Italy \\ ${ }^{8}$ I.N.F.N., Via Valerio 2, I-34100, Trieste, Italy \\ Received 2015 February 16; accepted 2015 June 5; published 2015 September 8
}

\begin{abstract}
The nature of the Type Ia supernova (SN Ia) progenitors remains uncertain. This is a major issue for galaxy evolution models since both chemical and energetic feedback plays a major role in the gas dynamics, star formation, and therefore the overall stellar evolution. The progenitor models for the SNe Ia available in the literature propose different distributions for regulating the explosion times of these events. These functions are known as the delay time distributions (DTDs). This work is the first one in a series of papers aiming at studying five different DTDs for SNe Ia. Here we implement and analyze the single-degenerate (SD) scenario in galaxies dominated by a rapid quenching of the star formation, displaying the majority of the stars concentrated in the bulge component. We find a good fit to both the present observed SN Ia rates in spheroidal-dominated galaxies and the $[\mathrm{O} / \mathrm{Fe}]$ ratios shown by the bulge of the Milky Way. Additionally, the SD scenario is found to reproduce a correlation between the specific SN Ia rate and the specific star formation rate (sSFR), which closely resembles the observational trend, at variance with previous works. Our results suggest that SN Ia observations in galaxies with very low and very high sSFRs can help to impose more stringent constraints on the DTDs and therefore on SN Ia progenitors.
\end{abstract}

Key words: galaxies: abundances - galaxies: evolution - galaxies: general - Galaxy: bulge - hydrodynamics methods: numerical

\section{INTRODUCTION}

Galaxy formation represents a multiscale, highly nonlinear process. The modeling of the observed galaxy populations with a self-consistent model-from the initial conditions left behind the big bang-requires knowledge of the coevolution of all the components of the universe. According to the $\Lambda$ cold dark matter paradigm, baryons condensate onto the dark matter halos, which constitute the sites of galaxy formation. The theories of galaxy formation aim at understanding at the same time the large-scale growth of the structure and small-scale processes such as the star formation from molecular clouds. This is a challenging task that can be tackled by using semianalytical or fully cosmological models. Both resort to recipes or subgrid modeling to include complex physical processes that cannot be numerically resolved. Hence, it is of utmost importance to confront the model results with observations to learn about galaxy formation and to test the validity of the adopted hypotheses.

Galaxy chemical evolution models provide us with a powerful tool to understand the way in which galaxies formed and evolved (Tinsley 1979; Matteucci \& Greggio 1986; Matteucci 1994). Since the information given by chemical abundances is the result of the nucleosynthesis production of the stellar populations and large-scale physics involved in the galaxy formation, chemical patterns can provide stringent constraints for galaxy formation models.

The treatment of chemical evolution with the inclusion of different models for Type Ia supernovae ( $\mathrm{SNe}$ Ia) opened the route to study galaxy formation by probing links between chemical patterns and relevant physical processes. These efforts are usually attempted with a variety of numerical techniques, such as multizone chemical evolution models (e.g., Matteucci \& Greggio 1986; Bravo et al. 1993; Chiappini et al. 2001; Pipino \& Matteucci 2004), semianalytic models of galaxy formation (e.g., Calura \& Menci 2009; Arrigoni et al. 2010; Jiménez et al. 2011; Yates et al. 2013; De Lucia et al. 2014; Gargiulo et al. 2015), and cosmological hydrodynamical simulations (e.g., Raiteri et al. 1996; Carraro et al. 1998; Mosconi et al. 2001; Nakasato \& Nomoto 2003; Kobayashi 2004; Tornatore et al. 2004; Nagashima et al. 2005; Scannapieco et al. 2005; Wiersma et al. 2009; Aumer et al. 2013; Few et al. 2014, to name a few). Nowadays, sophisticated computational tools and detailed observations of local and high-redshift galaxies enable us to improve galaxy formation models and develop more realistic schemes for the baryonic astrophysics.

One of the major astrophysical problems is the uncertain identity of the SN Ia progenitors. This is a matter of concern not only in galaxy formation theory but also in modern cosmology. The remarkable similarity shown by the light curves of the SNe Ia has made them excellent cosmological distance indicators. Hence, they are used to investigate the properties of dark energy and to test parameters of the cosmological model and the acceleration epoch of the universe (Perlmutter et al. 1999). However, given the still unknown nature of the progenitor systems of SNe Ia, systematic errors in the deduced distances based on calibrations of nearby $\mathrm{SNe}$ 
Ia could have an impact in the cosmological parameter estimation (Pan et al. 2012; Rest et al. 2013).

There is a general agreement in considering that core-collapse supernovae ( $\mathrm{SNe}$ II) are produced by massive short-lived stars $\left(M>8 M_{\odot}\right)$. The nucleosynthesis production of these events feeds the interstellar medium (ISM) with energy and, mainly, with the so-called $\alpha$-elements- $\mathrm{O}, \mathrm{Ne}, \mathrm{Mg}, \mathrm{Si}, \mathrm{S}$, and $\mathrm{Ca}$. On the other hand, the SNe Ia are the main contributor of the Fe in the universe (Greggio \& Renzini 1983; Matteucci \& Greggio 1986; Cappellaro et al. 1997). This element is usually considered to be a tracer of the metallicity in stars. Furthermore, the production and distribution of the chemical elements in the ISM affect the cooling rates of the gas (Sutherland \& Dopita 1993), the star formation processes, the subsequent stellar evolution (Pietrinferni et al. 2006), and the production of high-redshift dust (Maiolino et al. 2004; Bianchi \& Schneider 2007). Main properties such as the luminosity function and the mass distribution of galaxies might consequently be affected (White \& Frenk 1991; Scannapieco et al. 2005). By studying the enhancement of the $\alpha$ elements relative to $\mathrm{Fe}$, we expect to learn about the initial mass function (IMF) of the stellar populations and, very importantly, about the timescales in which $\mathrm{SNe}$ Ia become relevant. The time delay between core-collapse SNe and SN Ia injection of chemical elements in the ISM creates important patterns that can be used as clocks to tag particular events in the formation histories of galaxies. Given a starburst, SN Ia events occur following a distribution of explosion times that is known as the delay time distribution (DTD). Different scenarios for SN Ia progenitors produce different DTDs, which, in turn, create particular chemical patterns in the stellar populations and their host galaxies.

It has long been known (Hoyle \& Fowler 1960) that the explosion mechanisms, the chemical and energetic composition of the remnants, and the light curves of SNe Ia (with absence of $\mathrm{He}$ and $\mathrm{H}$ ) involve, at the most fundamental level, the combustion of a degenerate stellar core. Specifically, a white dwarf (WD) star of carbon (C) and oxygen (O) is led away from the equilibrium, following mass accretion and successive explosion. Among the two most popular scenarios for explaining $\mathrm{SNe}$ Ia based on a thermonuclear explosion of a $\mathrm{C}-\mathrm{O} \mathrm{WD}$, there is the single-degenerate (SD) scenario-a WD exceeding the Chandrasekhar mass through accretion from a nondegenerate companion star-where the mass accretion can assume many configurations. The secondary star is proposed to be a main-sequence star, a subgiant star, a helium star, or a red giant star (Whelan \& Iben 1973; Iben \& Tutukov 1984; van den Heuvel et al. 1992; Hachisu et al. 1996, 1999; Han \& Podsiadlowski 2004; Geier et al. 2013). In addition to the variety of possible secondary stars, some models include other effects such as the metallicity dependence proposed by Kobayashi et al. (1998). The authors found that when the metallicity is lower than $[\mathrm{Fe} / \mathrm{H}]<-1$, the winds developed by the primary star (WD) accreting mass through the Roche lobe are too weak and the explosion cannot occur.

The second most popular scenario involving a thermonuclear explosion of a $\mathrm{C}-\mathrm{O} \mathrm{WD}$ is the double-degenerate (DD) scenario-two WDs that lose angular momentum and energy by emitting gravitational waves and eventually merge. If they exceed the Chandrasekhar mass, they ignite as SNe Ia (Iben \& Tutukov 1984).

Other suggested theoretical scenarios include the "collisional scenario," where the head-on collision of two WDs occurs instead of the spiraling due to loss of gravitational wave radiation. These collisions of two WDs of sufficiently large masses are predicted in dense environments such as globular clusters and could explain $\mathrm{SNe}$ occurring in the nuclei of galaxies (Lorén-Aguilar et al. 2010). On the other hand, there is the "core-degenerate (CD) scenario," where a WD merges with the core of an asymptotic giant branch (AGB) star and forms a rapidly rotating WD. This new configuration has a mass close to and above the critical mass for explosion and was used recently as the best scenario to explain the observed properties of SN 2011fe (Soker et al. 2013). Additionally, the "double detonation" mechanism proposes a sub-Chandrasekhar WD accumulating a layer of helium-rich material on the surface sufficiently massive and degenerate to cause a detonation (Shen et al. 2013). Finally, another type of CD scenario considered is the "super-Chandrasekhar scenario." These models propose a Chandrasekhar- or super-Chandrasekhar-mass WD formed in the planetary nebula phase (or at the end of the common envelope phase), from a merger of a WD companion with the hot core of a massive AGB star (Tsebrenko \& Soker 2013). However, a common characteristic of all these scenarios is that similar nucleosynthesis processes occur during the explosion, creating mainly Fe. For a recent review on the $\mathrm{SN}$ Ia progenitors see Maoz et al. (2013).

Lately, many empirical DTDs have been proposed. The bimodal model by Mannucci et al. (2006) considers a DTD with two populations of progenitors of SNe Ia, one dominated by the "prompt" component that explodes within $100 \mathrm{Myr}$ after the formation of their progenitors, and the "tardy" component exploding on a wide period of time extending up to $10 \mathrm{Gyr}$. The shape of the bimodal distribution is given by the sum of two functions: a Gaussian centered at $5 \times 10^{7} \mathrm{yr}$, and an exponentially declining plateau function. Moreover, similar power-law DTDs $\left(\sim t^{-1}\right)$ have been recovered from different observational surveys. For instance, Totani et al. (2008) using field elliptical galaxies proposed such a power law. Maoz et al. (2012) also suggested a very similar DTD based on a sample of 132 SNe Ia from the Sloan Digital Sky Survey II. Finally, Pritchet et al. (2008) presented a power-law DTD $\left(\sim t^{-0.5}\right)$ from the SuperNova Legacy Survey (SNLS).

The DTD represents a powerful tool, not only for testing models and helping to constrain the progenitor SN Ia scenarios, but also for obtaining an accurate description of the chemical and energetic feedback from SNe Ia. The aim of our work is to test which is the best scenario for SNe Ia in the framework of cosmological simulations. In particular, we will include and analyze in detail, for the first time to our knowledge, five different DTDs for SNe Ia in a smooth particle hydrodynamical code. Our results will be presented in a series of three papers.

In this first paper we will discuss in detail the SD model and compare our results, obtained for a bulge-type galaxy, with the chemical abundances and SN Ia rates for this kind of galaxy. In particular, we will compare our results with the abundance data for the Milky Way (MW) bulge. We study global properties such as the correlation found by Sullivan et al. (2006) between the specific star formation rate (sSFR) and the specific SN Ia rate (SSNIaR). The reason for starting with this particular scenario is that it has been suggested as one the best models to reproduce the chemical properties of galaxies (Matteucci et al. 2006, 2009).

This paper is organized as follows: Section 2 describes the analytic DTD for the SD scenario. Section 3 briefly describes 
the main aspects of the numerical code and presents the initial conditions. In the following subsections we present the implementation of DTD in the code. In Section 4 we show the calibration of the model with the observables. In Section 5 we study the observed correlation found by Sullivan et al. (2006), between the SSNIaR and the sSFR for the SD scenario. The main conclusions are summarized in Section 6.

\section{THE SD SCENARIO}

In the SD scenario proposed by Whelan \& Iben (1973), SNe Ia originate from a binary system with one $\mathrm{C}-\mathrm{O}$ WD and a red (super) giant star. The WD accretes mass from the red giant through the Roche lobe, and an explosive nucleosynthesis process occurs when the WD reaches the Chandrasekhar mass. Calculations of the SN Ia rates were performed on the basis of this model (Greggio \& Renzini 1983; Matteucci \& Greggio 1986; Tornambe \& Matteucci 1987; Matteucci et al. 2009) for both our Galaxy and elliptical galaxies.

To calculate the SN Ia rates in the framework of the SD scenario is necessary to know the range of lifetimes from the stars belonging to the exploding binary systems. One way to do this is to evaluate the minimum and maximum masses allowed for these systems, taking into account the fact that SNe Ia occur in all galaxies, including ellipticals that do not show any active star formation. In this work we adopt the model of Greggio \& Renzini (1983, hereafter GR83), where the lifetimes of the stars exploding as $\mathrm{SNe}$ Ia are between the mass range of $0.8-8 M_{\odot}$. Then, according to this mass range, after a starburst the first systems to explode as an SN Ia (e.g., $8+8 M_{\odot}$ systems) will require no more than $\sim(3-4) \times 10^{7} \mathrm{yr}$. And this is the most massive system possible with a total mass of $16 M_{\odot}$. On the other hand, the minimum mass possible for a binary system giving rise to an SN Ia explosion is estimated to be $3.0 M_{\odot}$ (see GR83). This total minimum mass will ensure that the system can contain a WD and a companion massive enough to allow the WD to reach the Chandrasekhar mass after accretion.

To calculate the SN Ia rates, we follow the formulation of Matteucci \& Recchi (2001, hereafter MR01):

$$
\begin{aligned}
R_{\mathrm{Ia}}(t)= & A \int_{M_{\mathrm{B}, \text { inf }}}^{M_{\mathrm{B}, \text { sup }}} \phi\left(M_{\mathrm{B}}\right) \\
& \times \int_{\mu_{\min }}^{\mu_{\max }} f(\mu) \psi\left(t-\tau_{M_{2}}\right) d \mu d M_{\mathrm{B}}
\end{aligned}
$$

Here $M_{\mathrm{B}}=M_{1}+M_{2}$ is the total mass of the binary system (where $M_{1}$ is the primary and $M_{2}$ the secondary). In the limits of the integral we use the minimum and maximum total masses for the binary systems $\left(M_{\mathrm{B} \text {,inf }}\right.$ and $\left.M_{\mathrm{B} \text {,sup }}\right)$ described above. For more details see GR83 and MR01.

The only free parameter of this formulation is $A$, representing the fraction of binary systems able to produce $\mathrm{SNe}$ Ia in the mass range $3-16 M_{\odot}$. This parameter is generally fixed by reproducing the present-time observed SN Ia rate of the type of galaxy under study.

In Equation (1), the function $\psi(t)$ represents the SFR. This quantity needs to be evaluated at the time $\left(t-\tau_{M_{2}}\right)$, where $\tau_{M_{2}}$ accounts for the life of the secondary star. This constitutes the clock for the SN Ia explosion (Padovani \& Matteucci 1993). The function $\phi\left(M_{\mathrm{B}}\right)$ refers to the IMF from Salpeter (1955), defined in the mass interval $0.1-100 M_{\odot}$, with an index of $x=1.35$ :

$$
\phi\left(M_{\mathrm{B}}\right)=C M_{\mathrm{B}}^{-(1+x)}
$$

The mass fraction of the secondary star to the total mass of the system is the quantity $\mu=M_{2} / M_{\mathrm{B}}$, and its distribution function is given by $f(\mu)$. Previous studies (Tutukov \& Yungelson 1980) suggest a value of $\gamma=2$, which we also adopt here, as previously used in the formula

$$
f(\mu)=2^{1+\gamma}(1+\gamma) \mu^{\gamma} .
$$

It is worth noting that the integral in Equation (1) when computed without the SFR, namely, for an instantaneous starburst, is the so-called DTD function (shown for the SD scenario in the top panel of Figure 1).

This approach allows us to evaluate Equation (1), for each stellar particle with an SF episode, and estimate the rate of $\mathrm{SNe}$ Ia.

\section{THE NUMERICAL CODE}

We use an extended version of the Tree-PM smoothed particle hydrodynamics (SPH) code GADGET-3 (Springel 2005), which includes metal-dependent cooling (Sutherland \& Dopita 1993), star formation, chemical enrichment, SN feedback, and a multiphase model for the gas component (Scannapieco et al. 2006). In this scheme if two gas particles have dissimilar thermodynamic properties, they are explicitly prevented from being neighbors in the SPH calculations (unless they are in a shock), allowing the coexistence of gas clouds with different thermodynamical properties. The SN feedback scheme is coupled adequately with the multiphase model. As a result, the energy injection into the ISM produces the self-regulation of the SF and the triggering of mass-loaded galactic outflows. This SN feedback does not introduce mass-dependent parameters, as described in detail by Scannapieco et al. (2006). In this work, we assume that the total SN energy release in each event is $0.7 \times 10^{51} \mathrm{erg}$ and is equally distributed within the cold and hot phases surrounding a stellar particle. Our SN feedback model has already been used to study the formation of disk galaxies in a cosmological context (e.g., Tissera et al. 2013a, 2013b; Artale et al. 2015).

The cold and dense gas is transformed into stars when satisfying density and temperature criteria according to the Kennicutt-Schmidt law (Schmidt 1959; Kennicutt 1998). We adopt a Salpeter IMF, defined in the mass interval of $0.1-100 M_{\odot}$. SNe II are assumed to originate from stars more massive than $M>8 M_{\odot}$ and lifetimes of $\sim 10^{6} \mathrm{yr}$.

The SN Ia prescription included in Scannapieco et al. (2006) was originally proposed by Mosconi et al. (2001). Regarding the SN Ia implementations, the model of Scannapieco et al. (2006) will be referred to as the "original model" to distinguish it from the new implementations for the SNe Ia (based on different DTDs), presented in this and the following papers. In the original model the lifetimes of the binary system that explode as $\mathrm{SNe}$ Ia are assumed to be randomly distributed within a certain range given by $\tau_{\mathrm{SN} \text { Ia }}=[0.1,1] \mathrm{Gyr}^{9}$. This $\mathrm{SN}$

\footnotetext{
9 These limits can be varied as has been done by Scannapieco et al. (2009) in the Aquarius simulations, for example, thus considering a larger minimum lifetime for the distribution of the secondary mass, compared to the predictions of the SD scenario. This constitutes a simple approach based on the assumption that a fair fraction of SNe Ia will explode during a period $\tau_{\mathrm{SN} \text { Ia }}=10^{8}-10^{9} \mathrm{yr}$ (Greggio 1996). A fixed relative ratio between SNe II and SNe Ia is assumed, adopting an observationally motivated value (van den Bergh 1991), which can be associated with the free parameter $A$. The chemical yields in all the models are given by the W7 model of Iwamoto et al. (1999)
} 

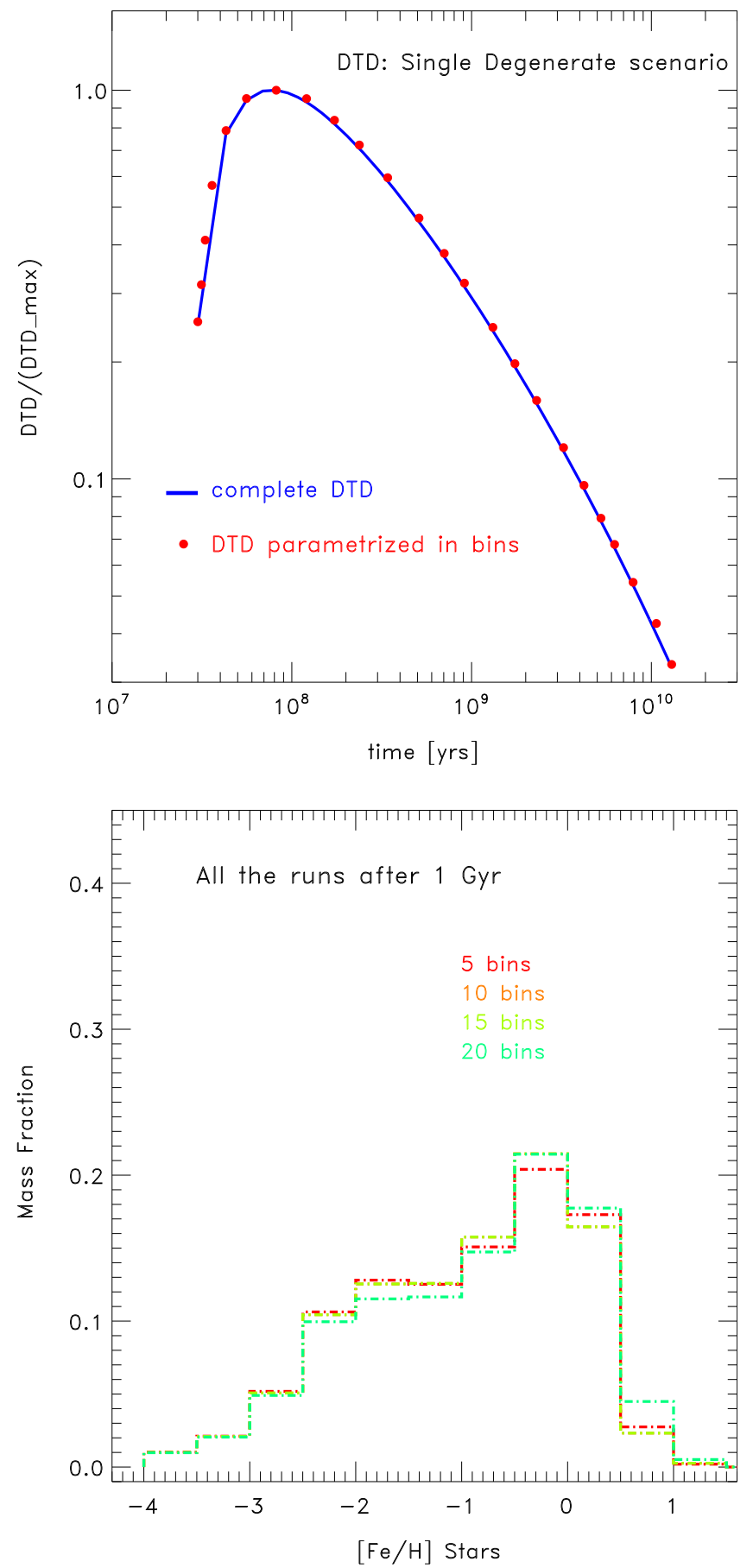

Figure 1. Top: full DTD for the SD scenario given by Matteucci \& Recchi (2001) and the DTD parameterized with 20 bins to reduce the computational costs (for more details, see the text). Bottom: distribution of $[\mathrm{Fe} / \mathrm{H}]$ ratios for the stars in the simulation, presented as an example of the relevant quantities of this study not being affected by the adopted number of bins used in the parameterization of the SD scenario.

Ia scenario, albeit simple, has been successful at reproducing many observational chemical patterns and trends, as shown in Tissera et al. $(2012,2013 \mathrm{~b}, 2014)$. The original model has the large advantage of being computationally inexpensive while grafting the main features of SNe Ia. However, there is room for improving the SN Ia modeling, as explained in the introduction. This would be of great importance when large ongoing or planned surveys of our Galaxy or nearby galaxies start to yield detailed abundances of the stellar populations such as APOGEE (a part of the Sloan Digital Sky Survey III; Anders et al. 2013), the GIRAFFE Inner Bulge Survey (Zoccali et al. 2014), the Calar Alto Legacy Integral Field Area survey (Sanchez et al. 2013), and the Sydney-AAO Multi-object Integral field spectrograph (SAMI; Croom et al. 2012), among others.

\subsection{The Simulated Galaxies}

We analyze the performance of the DTDs in simulations of pre-prepared galaxies in isolated dark matter halos. This IC is simple enough to highlight the effects of the different DTDs without being distracted by additional processes such as mergers and gas infall, which complicate the picture in fully hierarchical scenarios for galaxy formation. This simple approach allows us to more easily test the influence of the free parameter of the implemented DTDs.

The IC consists of a dark matter potential with an initial distribution following a Navarro-Frenk-White density profile (Navarro et al. 1997), which is let to evolve self-consistently with the baryonic component, with a concentration of $c=9$, an old stellar bulge with a Hernquist profile, and a old stellar exponential disk. The virial mass of this system is $M_{200} \sim$ $10 \times 10^{11} M_{\odot}$, with $10 \%$ of this mass in the form of baryons. The adimensional spin parameter is $\lambda=0.044$.

Initially, the gas is distributed in the disk component and represents $\sim 65 \%$ of the total baryonic mass of the galaxy. Within the total disk component, the gas represents $\sim 90 \%$ of the initial mass of the disk. This initial large gas fraction has been chosen to mimic a galaxy in its first stages of evolution in a simple way. At the beginning of the simulations the disk component is in equilibrium within the potential well $(Q>1)$ and has a scale length of $\sim 3.4 \mathrm{kpc}$. The gaseous disk has a lower $Q$ value (closer to $\sim 1$ ) and becomes unstable, developing clumps and bar instabilities. These two perturbations transport material into the inner regions of the galaxy, contributing to the formation of the bulge component. In particular, the bar drives gas inflows, which trigger important starbursts (for more details see Perez et al. 2011, 2013).

In all the simulations the initial number of baryonic particles is $\sim 80,000$ and that of the dark matter particles is $\sim 100,000$. Specifically, the initial gas particle is $\sim 7 \times 10^{5} M_{\odot}$, while the dark matter particle is $9 \times 10^{6} M_{\odot}$ and the stellar mass particle is $\sim 3 \times 10^{5} M_{\odot}$. The softening length for the gas particles is $200 \mathrm{pc}$, and for the dark matter particles we adopt $450 \mathrm{pc}$. The SFR efficiency is set at $c=0.1$. We follow the evolution of the systems until the gas to form stars is depleted and the star formation ceases.

We decomposed the bulge and disk components of the simulated galaxies by adopting a dynamical criterion based on the ratio between the rotational and dispersion velocities (e.g., Scannapieco et al. 2008). From this analysis we identify a bulge and disk component. The bulge is formed by a dissipative-dominated component and a central bar. The stellar density profiles show that the bulge is concentrated within the inner $\sim 3 \mathrm{kpc}$. This configuration shows a ratio of $B / T \sim 0.84$. Hence, the new stars formed for which we follow the chemical abundances are mainly part of the bulge.

Even more, the analysis of the SFR of the bulge shows that the stars formed during the strong starbursts, where the cold gas is exhausted within $\sim 1$ Gyr. The new stars remain concentrated within $\sim 3 \mathrm{kpc}$. All the simulations show this strong initial SF 
burst ending before $\sim 1$ Gyr owing to the gas depletion into stars and the effects of SN feedback, which ejects part of the gas (see subsection 4.1). The B+D stellar mass is nearly the same for all the simulations, $\sim 3.5 \times 10^{10} M_{\odot}$, typical of small ellipticals or spiral galaxy bulges.

By comparing these SF histories with the ones given by the model of Pipino \& Matteucci (2004, hereafter PM04), we conclude that the SF history of the gas component resembles spheroidal-type systems. This is consistent with the fact that the gas becomes unstable and collapses into the central region, feeding the strong starbursts. Most of the new stars are centrally concentrated, contributing to the formation of the bulge component. Therefore, we compare the present-day SN Ia rates with those observed in spheroidal-dominated galaxies. The chemical patterns will be confronted with observations of the Galactic bulge as these are the only observations available of individual stars in a bulge. We do not attempt to reproduce exactly these data since the formation history of the Galactic bulge seems to be more complicated (Rojas-Arriagada et al. 2014), but to use the data to set global constraints on the $A$ parameter.

\subsection{Implementation of the DTD}

The numerical code estimates whether a given gas particle meets the condition to be transformed into stars. Then, the code follows over time the new stellar particle, representing a single stellar population, and calculates the number of SNe Ia that should be produced as a function of time, according to the assumed DTD. The free parameter $A$ is adjusted to reproduce the present-time observed SN Ia rates. Analytical or semianalytical models fix the value of $A$ requiring the galaxy to reproduce the observed $\mathrm{SN}$ Ia rates according to its morphology. In a numerical simulation, $A$ is tuned at a particle level, so a given stellar particle does not have any a priori knowledge of the morphology of the galaxy it inhabits. On the contrary, reproducing the observed $\mathrm{SN}$ Ia rate according to morphology should be a prediction of our models. Note that in our models, gas and star particles will evolve according to the physical laws in a nonlinear way. Hence, reproducing the observed values is a challenge, even with an IC as simple as the one adopted in this work.

As mentioned in the Introduction, there are several theoretical scenarios for the DTDs. This paper considers the SD scenario by MR01 and leaves for Paper II the following scenarios: the DD scenario by Greggio (2005), the bimodal scenario by Mannucci et al. (2006), and the power laws proposed by Maoz et al. (2012) and by Pritchet et al. (2008). The implementation of the DTDs is the same for all the models.

The drawback of including detailed descriptions of the DTDs in the simulations is the computational cost for our particular $\mathrm{SN}$ feedback model. The objective is indeed to keep the model running efficiently to allow its use in large-scale cosmological simulations. Therefore, solving the integrals given by Equation (1) for each particle, at each time, is highly inefficient. This is related to the methodology followed by the SN feedback model. In the adopted scheme, the code has to search for the nearest hot and cold gas particle neighbors of a given stellar particle in order to inject the energy and chemical elements. This procedure is very time-consuming. To alleviate this problem, we include the DTDs in the simulations by creating tables for each DTD. We use 20 equally time-spaced bins to parameterize the complete DTD distributions. This can be visualized in the top panel of Figure 1, where we show the complete DTD for the SD scenario given by MR01 (solid line) and the resampling with 20 intervals or bins (red circles). The representation of the DTD with this lower number of intervals reduces the computational costs by limiting the hot and cold neighbor searches required to inject the $\mathrm{SN}$ Ia products, without losing the relevant information stored in the complete DTD distribution. The SN Ia rates for each stellar particle (i.e., representing a single stellar population) are estimated by searching in the DTD table for the number of SNe Ia that should be produced according to the particle stellar age and the adopted progenitor model. Then, the total SN Ia rates for the central spheroid are calculated by adding the contributions from each star particle identified to belong to this component. In particular, we use the rates at the time when the SFR is quenched to compare with other models and observations. Thanks to this efficient implementation based on pre-prepared tables for the DTDs, the computational costs are significantly reduced.

We study the convergence of different parameterizations of the DTDs varying the bins numbers used to map the DTDs. We run the SD-1 model with $[5,10,15,20]$ bins for fitting the DTD curve, finding that the distribution of metals, the SFR, the specific SFR, the SN Ia rates, and other relevant quantities for this study do not not change significantly with the number of bins chosen. To illustrate this fact, we show in the bottom panel of Figure 1 histograms of the mass fraction of stars within a given $[\mathrm{Fe} / \mathrm{H}]$, calculated for models with different numbers of bins. It can be seen that the differences between the fractions of mass are negligible. Notice, for example, that the fraction of stars with $[\mathrm{Fe} / \mathrm{H}]>0$ varies less than $0.2 \%$ between the models.

\section{CALIBRATING THE SD SCENARIO}

Chemical evolution models calibrate the DTDs using the free parameter $A$ fixed a posteriori, to fit the present-day observed rate of SNe Ia. This parameter accounts for the fraction of binary systems that undergo an SN Ia event in a starburst once the IMF is fixed. In the SPH simulations $A$ is also a free parameter but on a particle basis. Thus, it is not possible to know a priori which morphological type of galaxy a given particle inhabits, particularly in a cosmological simulation. The parameter $A$ has to reflect the underlying physics and, at the same time, be able to reproduce the mean observable constraints.

To explore the range of $A$ values able to reproduce observations, we make use of the multizone chemical evolution model of PM04 for bulge-like systems of similar mass and SF history to the SPH galaxies. Once we find a parameter space for $A$, we run the SPH simulations. The advantage of this methodology is the small computational cost of running the multizone chemical evolution model of PM04, compared to the cost of running several SPH simulations until selecting the proper values for $A$. Caution should be taken since a change in $A$ might produce a nonlinear response in the SPH simulations, as the SN feedback and gas metallicities will also change, modifying the cooling rates and the availability of cold gas for subsequent star formation activity.

Specifically, in the PM04 model a galaxy is divided into several noninteracting shells. The chemical evolution equations are solved in each shell in order to reproduce the evolution of the elemental abundances. The SFR is given by the law $\psi$ 
Table 1

Main Characteristics of the Simulated Galaxies Run with the SD Scenario and Varying $A$

\begin{tabular}{llcl}
\hline \hline Model & \multicolumn{1}{c}{$A$} & $\begin{array}{c}\langle\text { SFR }\rangle \\
\left(M_{\odot} \mathrm{yr}^{-1}\right)\end{array}$ & $\begin{array}{c}\text { SN Ia Rates } \\
\left(\mathrm{N} / \mathrm{yr}^{-1}\right)\end{array}$ \\
\hline SD-1 & 0.0015 & 83 & 0.002 \\
SD-2 & 0.00015 & 60 & 0.00006 \\
SD-3 & 0.0075 & 84 & 0.0080 \\
SD-4 & 0.00075 & 76 & 0.0016 \\
Original & 0.0015 & 53 & 0.0027 \\
\hline
\end{tabular}

Note. The mean observed SN Ia rate for spheroidal-dominated galaxies of stellar mass $\sim 3.5 \times 10^{10} M_{\odot}$ is $\sim 0.0017 \mathrm{SNe}$ per year (Li et al. 2011).

$(t)=\nu \rho_{\text {gas }}(t)$, assumed to be proportional to the gas density $\rho_{\text {gas }}$, via a constant $\nu$, representing the star formation efficiency. This quantity in the PM04 models increases with the baryonic galaxy mass. For the comparison with the SPH simulations, we run the model of PM04 adopting $\nu=50 \mathrm{Gyr}^{-1}$ for a galaxy with initial mass of $10^{11} M_{\odot}$ and an infall time of $0.01 \mathrm{Gyr}$. Because of the stellar winds suffered through its evolution, the galaxy ends up with a final mass of $\sim 4.7 \times 10^{10} M_{\odot}$. And this mass is of the order of magnitude of the new stellar mass formed in the SPH galaxies $\left(\sim 3.5 \times 10^{10} M_{\odot}\right)$. The SFR histories of the SPH galaxies and the PM04 galaxies are very similar, showing a bursty behavior at the beginning with a fast quench after $\sim 1$ Gyr. These SFRs are shown in the top panel of Figure 2.

The following step is to use the SFR of the SPH galaxy obtained running the original model of Scannapieco et al. (2006) as an input to the model of PM04. In this way, we obtain a prediction for the rates of SNe Ia and $\mathrm{SNe}$ II for the given galaxy. By varying the value of $A$ in the PM04 model, we can find the adequate parameter space for $A$. This parameter space is then the one we adopt in the SPH simulations. As a constraint to our models we use the rate-size relation of $\mathrm{Li}$ et al. (2011) and derive the observed SN Ia rate at the present time, following the method shown by Valiante et al. (2009). We do this for a galaxy with baryonic mass similar to our simulated galaxy. Thus, for a system of $\sim 3.5 \times 10^{10} M_{\odot}$, the rate predicted by $\mathrm{Li}$ et al. (2011) is $\sim 0.0017 \mathrm{SNe}$ Ia per year.

We run four experiments with the same IC and the SD model using $A=[0.0015,0.00015,0.0075,0.00075]$ (SD-1, SD-2, SD-3, and SD-4 models). Larger or smaller values are strongly ruled out by the PM04 model. Table 1 summarizes their main properties, including the predicted SN Ia rates (column 4), calculated when the SFR is quenched. It can be seen that we obtain good agreement with SD-1 and SD-4. The original model, with $A=0.0015$, also reproduces the observed SN Ia rates.

The behavior of the SN Ia rates as a function of time for the different models is shown in Figure 3. It becomes clear from the plot that the rates are affected not only by the $A$ parameter but also by the lifetimes of the progenitor systems that explode as SNe Ia given by each model. Recall that for the SD scenario the first systems made of two $8 M_{\odot}$ stars explode $\sim(3-4) \times 10^{7}$ yr after the starburst. On the other hand, for the original model the lifetimes are assumed to be randomly distributed within a certain range given by $\tau_{\mathrm{SN} \text { Ia }}=[0.1,1] \mathrm{Gyr}$. Thus, the predicted rates for the original model are delayed in time relative to those of the SD scenario, and lacking the prompt $\mathrm{SNe}$ Ia (those exploding in the first $0.1 \mathrm{Gyr}$ ), as can be
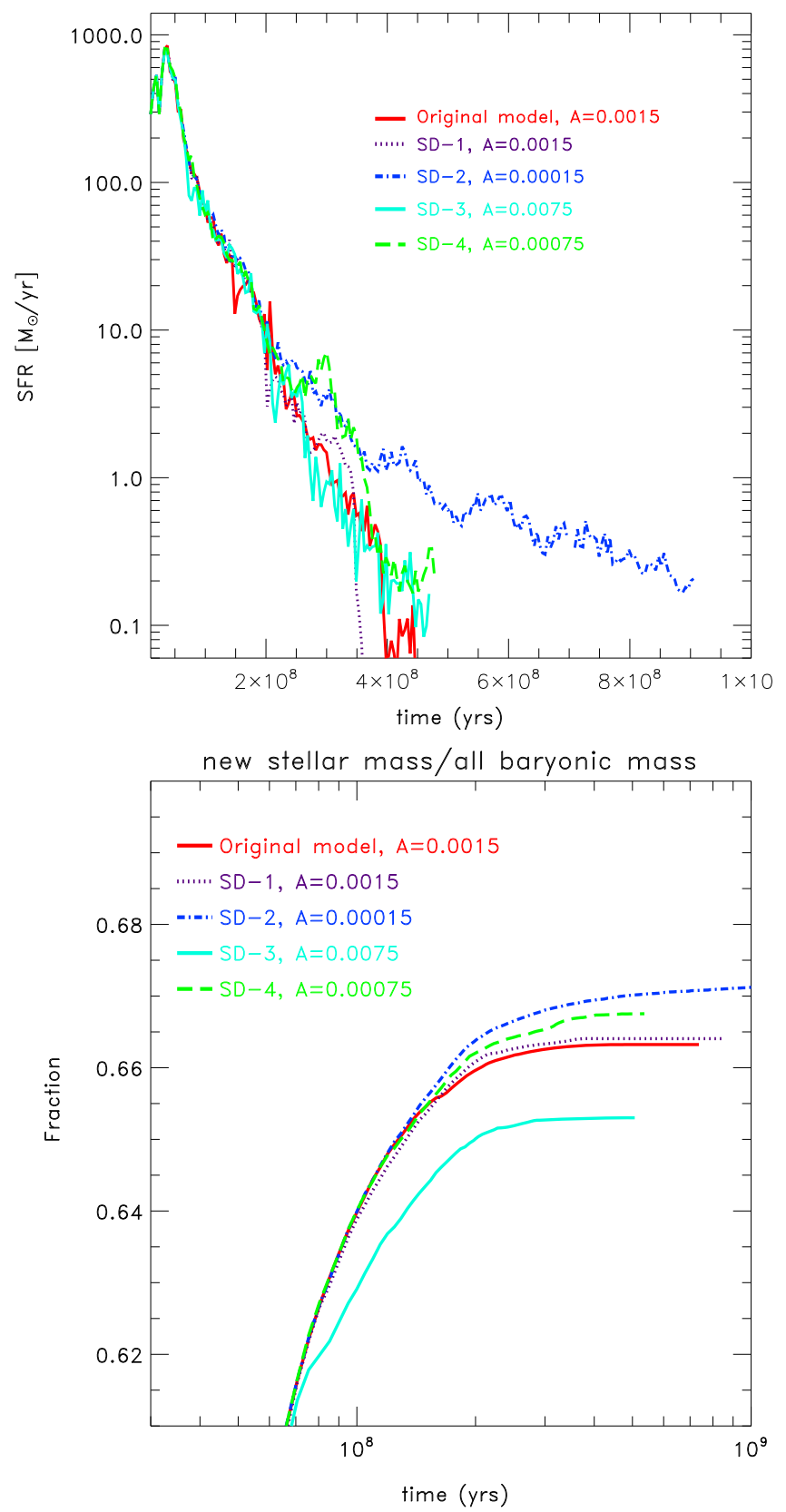

Figure 2. SN Ia rates for all the models as a function of time.

seen in Figure 3. In the following section we analyze the consequences of this delay in the SFR.

\subsection{Star Formation Rate}

In Figure 2 (top panel), we show the SFR of the simulated galaxy for all the explored $A$ values. The SFRs are estimated by using constant time intervals along the history of the galaxies. In all the runs, there is a strong burst at the beginning, followed by a decay of the SFR until the gas is exhausted. Since the simulated galaxy is isolated, there is no external gas reservoir to keep feeding the SF. No meaningful differences between the models appear until the SFR declines below $\sim 10 M_{\odot} \mathrm{yr}^{-1}$. The SFR for the SD-1 model is the first to drop to $\sim 0.01 M_{\odot} \mathrm{yr}^{-1}$ after $\sim 4 \times 10^{8}$ yr. Model SD-2, with the lowest $A$ value, is the last to exhaust the gas by forming stars after $\sim 8 \times 10^{8} \mathrm{yr}$. 


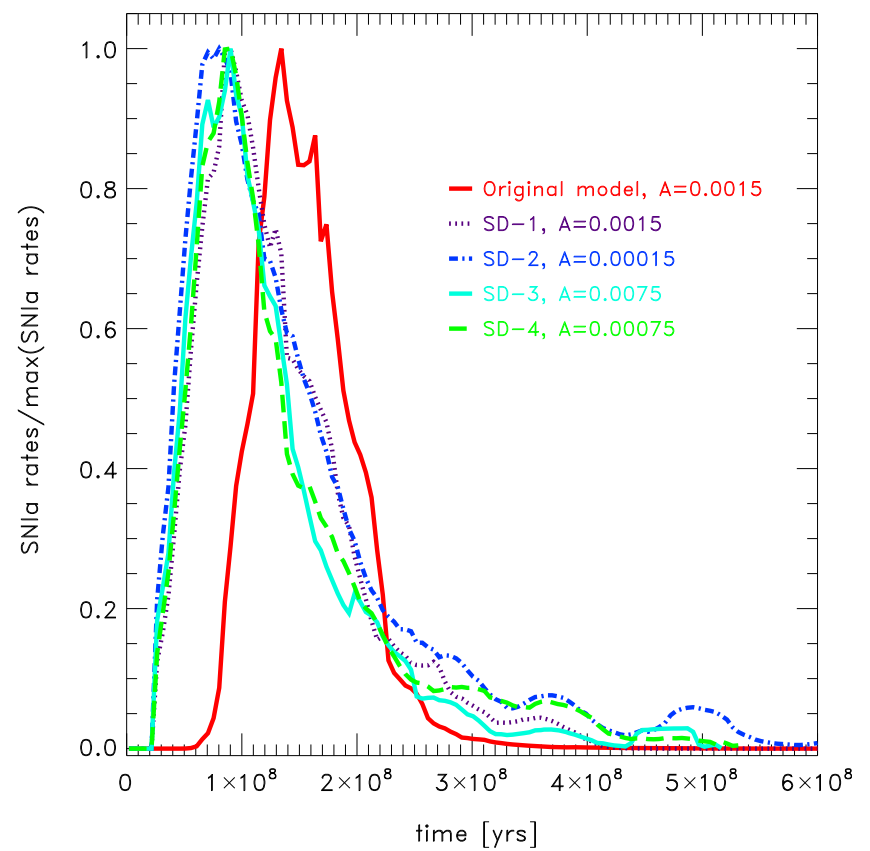

Figure 3. SFR history (top panel) and mass fraction of newborn stars (bottom panel) for the SPH galaxies run with the same IC and varying the $A$ values.

Model SD-1 and the original model share the same value of $A$ but show different quenching times, the latter being the one with more extended SFR. All the simulations have their SFR quenched before $1 \mathrm{Gyr}$, owing to gas depletion into stars and/or the effects of SN feedback, which heats up part of it.

Alternatively, we quantify the effects of the parameter $A$ acting on the regulation of the SF by the estimation of the mass fraction of newborn stars. Figure 2 (bottom panel) shows clearly how different $A$ can regulate the SF activity according to the total energy that $\mathrm{SNe}$ Ia inject into the ISM. The efficiency of the stellar production depends on the available cold gas in galaxies, a quantity linked to the number of SN events occurring in the galaxy. From Figure 2 we can see that when the value of $A$ increases, the fraction of new stars decreases accordingly. This can be clearly appreciated from the comparison between the final fractions reached in SD-3 and SD-2, with the highest and lowest values of $A$, respectively. However, the variations between models are very small (less than $2 \%$ ), indicating that, at least for this IC, the effects on the regulation of the SF are minor, if sensible values of $A$ are assumed.

Comparing the original and SD-1 models, we can see that they produce the same fraction of newborn stars, although the star formation activity is quenched earlier in the SD-1 model. Recall that in the SD model the minimum lifetime of SNe Ia is $\sim 3 \times 10^{7} \mathrm{yr}$, whereas in the original model it is $\sim 1 \times 10^{8} \mathrm{yr}$ (see Figure 3). This explains the differences in the SFR quenching times of the two models. Notice that the DTD implementation seems not to affect the efficiency of the fraction of newborn stars formed in the galaxies when compared to the original model. Observational evidence of the existence of prompt $\mathrm{SNe}$ Ia strongly argues in favor of a minimum timescale as long as $\sim 10^{8}$ yr (Bonaparte et al. 2013, and references therein).

Notice that even when the SNe Ia can continue to pollute the ISM with the nucleosynthesis production after the SFR has stopped, this will not affect the chemical abundances of the stellar populations since no new stars will be formed from the enriched gas. Therefore, in the following section we analyze the distribution of chemical elements at this particular time: when the SFR is quenched. Analytical and multizone chemical evolution models adopt a similar criterion, which we also chose here for the sake of comparison.

\subsection{The $[\mathrm{O} / \mathrm{Fe}]$ versus $[\mathrm{Fe} / \mathrm{H}]$ Diagram}

We study here the predicted $[\mathrm{O} / \mathrm{Fe}]$ versus $[\mathrm{Fe} / \mathrm{H}]$ relation by each of the models of the SD scenario included in the SPH simulations. It is worth remembering that the parameter $A$ is tuned to reproduce the present-time observed SN Ia rate. In our case, the values of $A$ that best reproduce the present-time rate are those of SD-1 and SD-4 models, as already shown in Section 4 . Here we test the effect of varying $A$, namely, the fraction of $\mathrm{SNe} \mathrm{Ia}$, on the predicted $[\mathrm{O} / \mathrm{Fe}]$ ratios. ${ }^{10}$

We interpret the evolution of the abundance ratios of $[\mathrm{O} / \mathrm{Fe}]$ versus $[\mathrm{Fe} / \mathrm{H}]$ with the help of the time-delay model of Tinsley (1979). It is expected that the delay in the production of Fe by SNe Ia ejected into the ISM-in relation to the rapid production of $\alpha$-elements by $\mathrm{SNe}$ II-leaves a characteristic signature in the $[\mathrm{O} / \mathrm{Fe}]$ versus $[\mathrm{Fe} / \mathrm{H}]$ diagram MR01. The ejection of $\mathrm{Fe}$ from $\mathrm{SNe}$ Ia is regulated by the DTD. The main effect of the delayed Fe production in relation to the $\alpha$-elements produced by $\mathrm{SNe}$ II is to create an overabundance of $\alpha$-elements. In particular, the oxygen remains high until SNe Ia become important and the ratio $[\mathrm{O} / \mathrm{Fe}]$ starts to decline. This point is identified by a "knee" in the $[\mathrm{O} / \mathrm{Fe}]$ versus $[\mathrm{Fe} / \mathrm{H}]$ diagram (see Figure 4).

Assuming that bulges and elliptical galaxies experience a strong burst of star formation lasting a short time (less than 1 Gyr), Matteucci \& Brocato (1990) predicted that $[\alpha / \mathrm{Fe}]$ should be supersolar for a large interval of $[\mathrm{Fe} / \mathrm{H}]$. In fact, objects such as bulges that evolve very fast with an intense SFH quickly reach solar metallicity only as a result of the production of Fe by SNe II. When SNe Ia start to explode, the production of $\mathrm{Fe}$ is enhanced and a change in the slope (the knee) in the $[\mathrm{O} / \mathrm{Fe}]$ versus $[\mathrm{Fe} / \mathrm{H}]$ diagram occurs at $[\mathrm{Fe} / \mathrm{H}] \geqslant 0$.

We compute the average stellar mass abundance of $[\mathrm{Fe} / \mathrm{H}]$ and $[\mathrm{O} / \mathrm{Fe}]$ for the stars in the bulge (see Section 3.1), for each of the models. The ratios $[\mathrm{Fe} / \mathrm{H}]$ and $[\mathrm{O} / \mathrm{Fe}]$ are calculated by adding the masses of the corresponding chemical elements stored in the star particles identified to belong to the central spheroid. We show the SD models with different values of $A$ in Figure 4, where the different colored lines refer to each of the models compared to Galactic bulge stars. A sample of dwarfs and subgiant stars from Bensby et al. (2013) and red giant stars from Lecureur et al. (2007) are considered for comparison.

Figure 4 shows the observed data points lying in between the curves displayed by models SD-3 and SD-2, corresponding to the highest and lowest values of $A$, respectively. However, model SD-2 predicts a too flat $[\mathrm{O} / \mathrm{Fe}]$ ratio. This means that the fraction of SNe Ia is too low. On the other hand, model SD-3 is an extreme case where there are too many $\mathrm{SNe}$ Ia, and it predicts, in fact, a continuous decrease of the $[\mathrm{O} / \mathrm{Fe}]$ ratio, at odds with the observations. The data are best represented by the models SD-4 and SD-1. The former model (SD-4) fits the zero point of the data following the observed trend up to $[\mathrm{Fe} /$

\footnotetext{
${ }^{10}$ In the original and SD models the yields used for SNe II are the metaldependent ones from Woosley \& Weaver (1995). For the production of SNe Ia we assume the W7 model by Iwamoto et al. (1999).
} 


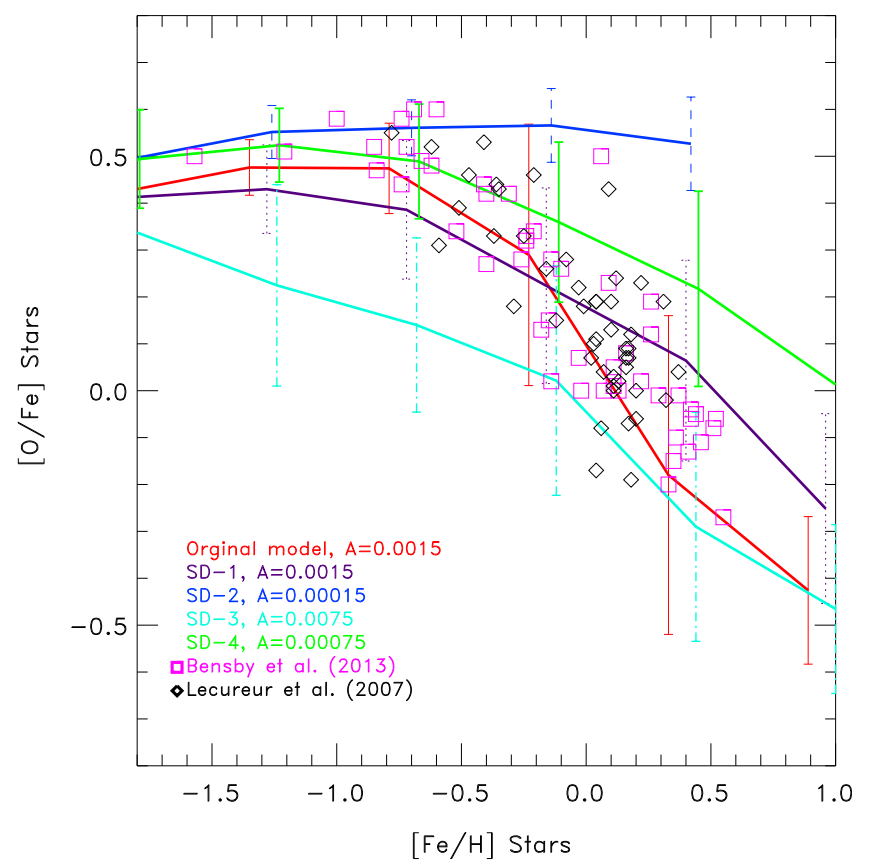

Figure 4. $[\mathrm{O} / \mathrm{Fe}]$ vs. $[\mathrm{Fe} / \mathrm{H}]$ exhibited by the bulge stars in the $\mathrm{SPH}$ simulated galaxies with the SD scenario by Matteucci \& Recchi (2001) and varying $A$. These models are compared with observational $[\mathrm{O} / \mathrm{Fe}]$ ratios for stars in the Galactic bulge by Lecureur et al. (2007) and Bensby et al. (2013). See Table 1 for details on the simulations.

$\mathrm{H}] \sim-0.25$. Meanwhile, SD-1 matches the slope and passes through the data better. These models predict a long plateau for the $[\mathrm{O} / \mathrm{Fe}]$ ratio and a knee occurring at high $[\mathrm{Fe} / \mathrm{H}]$, as observations suggest. However, as can be seen in Figure 4, at high metallicity the slopes of both models do not so nicely follow the observed $[\mathrm{O} / \mathrm{Fe}]$ ratios. This is perhaps a consequence of yields adopted in this work. The WW95 yields do not include mass loss from massive stars, which is particularly important for Wolf-Rayet stars and for supersolar metallicity. Its effect is to increase the yields of carbon and helium and to depress that of oxygen, as extensively described in McWilliam et al. (2008). Notice that SD-1 and SD-4 models also fit the present-time $\mathrm{SN}$ Ia rates (see Section 4). The original model, albeit simple, fits the data in the whole range.

From the previous analysis, we conclude that the chemical enrichment shown by the $[\mathrm{O} / \mathrm{Fe}]$ ratios changes linearly with $A$ in the SD scenario. Therefore, it is possible to find a range of values of $A$ that can predict the current $\mathrm{SN}$ Ia rates and the expected trend for $[\mathrm{O} / \mathrm{Fe}]$ ratios at the same time. It also shows the importance of calibrating the models using observables.

The $[\mathrm{O} / \mathrm{Fe}]$ versus $[\mathrm{Fe} / \mathrm{H}]$ ratios observed in the solar neighborhood had been reproduced for MW-type galaxies by several galaxy formation models (e.g., Calura et al. 2012; Yates et al. 2013; Few et al. 2014; De Lucia et al. 2014), although some of them fail to reproduce the most enriched stars for the MW bulge, showing an offset with the data (see, e.g., Figure 12 from De Lucia et al. 2014).

The "knee" of the bulge shown in Figure 4 occurs around $[\mathrm{Fe} / \mathrm{H}] \sim-0.25$, at variance from the observed value for the disk component of the $\mathrm{MW}$ found at $[\mathrm{Fe} / \mathrm{H}] \sim-1$ (François et al. 2004). This difference is a consequence of the strong SF experienced by the spheroidal component, making it evolve faster than the disk. As a result, it reaches higher values of $[\mathrm{Fe} /$ $\mathrm{H}]$ at the time when the SN Ia star become important and restore the bulk of Fe to the ISM (a prediction from chemical evolution models; Brocato et al. 1990). Therefore, we remind the reader that the timescale for the $\mathrm{SN}$ Ia reaching its maximum enrichment, usually quoted as $\sim 1 \mathrm{Gyr}$, is not universal but only valid for the solar neighborhood, as can be seen in this example and was pointed out by MR01.

\section{THE CORRELATION BETWEEN THE SN Ia RATES AND THE SPECIFIC SFR}

Observations relating the SN Ia rates of galaxies to the characteristics of the host galaxy such as their morphology, colors, and SFR are powerful constraints to our models. Consequently, in this section we compare the results of the best SD scenario with observations presented by Sullivan et al. (2006). In that paper, the authors found a correlation between the sSFR (the SFR per unit mass of the galaxy) and the SSNIaR (the rate of SNe Ia per unit of galaxy mass) for galaxies in the SNLS galaxy sample. This correlation is based on a sample of 100 spectroscopically confirmed SNe Ia, plus 24 photometrically classified events, distributed over $0.2<z<0.75$. The stellar masses and SFRs for the SN Ia host galaxies are estimated by fitting their broadband spectral energy distributions (SEDs) with the galaxy spectral synthesis code PEGASE.2 (Fioc \& Rocca-Volmerange 1997). They adopt the IMF of Kroupa (2001). The sSFR for the sample is measured as the ratio between the mean SF rate over the past $0.5 \mathrm{Gyr}$ and the current stellar mass of the galaxy, resulting from the SED fitting. They choose this interval of time to avoid systematic errors for galaxies for which the redshift is not known.

Furthermore, Sullivan et al. (2006) compared the correlation with observations of a morphologically classified sample of SN Ia host galaxies in the local universe presented by Mannucci et al. (2005). It became clear from this comparison that the sample of galaxies in the local universe shares the same trend displayed by SNLS galaxies at higher redshift. Therefore, the SSNIaR is a function of the host galaxy sSFR, with strongly star-forming galaxies hosting roughly 10 times as many SNe Ia per unit mass than passive galaxies with no star formation.

Moreover, Smith et al. (2012) obtained the SN Ia rates of galaxies with different SFR activity located at intermediate redshift $(0.05<z<0.25)$, from a sample of 342 galaxies belonging to the Sloan Digital Sky SuperNova Survey II (SDSS-II SN Survey). The authors estimated the host stellar masses and the recent SFRs using the code PEGASE.2. They confirmed the existence of a correlation between the SSFR and the SSNIaR. Therefore, the correlation holds for intermediate redshifts (Sullivan et al. 2006) and for the local universe (Mannucci et al. 2005), indicating no evolution with redshift within the range of $0.05<z<0.75$.

Sullivan et al. (2006) concluded that the correlation is difficult to reconcile with a model for $\mathrm{SNe}$ Ia that originates solely from an old evolved stellar population. Instead, they proposed a scenario of two separate components: a prompt component with a short delay time and an old component with a long delay time, consistent with the bimodal model of Mannucci et al. (2006). Here we explore this relation with the SD model, and in Paper II the analysis will be extended to the other DTD models.

To assess whether our best SD models are able to reproduce a correlation such as the observed one, the SSNIaR and the sSFR are estimated as the galaxy evolves, under the hypothesis 
that observations might catch galaxies at different stages of evolution (see also Figure 12 in Greggio 2010 for a similar approach using analytical models). For this purpose, we calculate the SSNIaR and the sSFR of the simulated galaxies as a function of time. This is done by using the SFR histories and the SN Ia rates of the simulated galaxies and their corresponding stellar masses as a function of time (e.g., Figure 2). These quantities allow us to calculate the mean sSFR and SSNIaR at different stages of the galaxy evolution. The averages over the whole stellar population are taken at a certain period of time, chosen to be within $0.5 \mathrm{Gyr}$, in order to mimic observations. ${ }^{11}$ Smith et al. noticed that the zero point of the correlation is affected by the choice of the IMF, systematic uncertainties concerning the accuracy of the derived properties of host galaxies, and the photometric redshift estimates produced by the PEGASE SED fitting code. Therefore, because of all these uncertainties, we focus on the study of the slope of this correlation and prefer not to use the error bars provided by the observational works. Consequently, the simulated relations are normalized by an ad hoc factor to simplify the qualitative comparison of the observed slope of the correlation.

A combination of the observed data by Mannucci et al. (2006), Sullivan et al. (2006), and Smith et al. (2012) and the results for the SD-1 and SD-4 models are shown in Figure 5. Although a word of caution is necessary when making comparisons between the evolution in time of single objects - like our simulated galaxies - and observations referring to a mix of different objects that might have undergone different evolutionary histories, it is interesting that our model including the SD scenario reproduces a clear correlation that agrees remarkably well with observations.

As can be seen from Figure 5, at the beginning the simulated correlations follow a different trend, so that the SSNIaR increases abruptly to high values of sSFR before reaching the expected observed trend. This feature is detected for all $A$ values in the SD model, as can be seen in models SD- 1 and SD4. These strong variations in the SSNIaR are produced in a short interval of sSFR for most of the models $(8.5<\log$ sSFR $<7.5)$ and are related to the time delay in the SN Ia explosions.

To understand the physical reasons causing the sSFRSSNIaR correlation, we plotted the logarithm of sSFR and the SSNIaR as a function of time for the SD-1 and SD-4 models in Figure 6. The sharp increase of SSNIaR for high sSFR reflects the onset of an SN Ia and how quickly it reaches the maximum value. After that, both the SSNIaR and the sSFR decrease, establishing the observed correlation. For very low sSFR, there is still a residual SSNIaR, as the generation of these events is delayed in time and the correlation is lost.

The particular feature seen at the beginning of the sSFRSSNIaR relation is not shown by the current observational data and represents a prediction from the simulations. If these early stages could be observed, then they could be used to set limits on the shape of the DTDs. Of course, the system we have used to explore the effects of varying the DTDs is very simple. The SF history of galaxies could certainly be more complicated with several starbursts occurring during their life. The analysis of these complex stellar populations is delayed to a forthcoming paper using cosmological simulations.

\footnotetext{
11 To correct for the different IMFs used in this work (Salpeter) and the observations (Kroupa), we adopt the transformation suggested by Longhetti \& Saracco (2009).
}

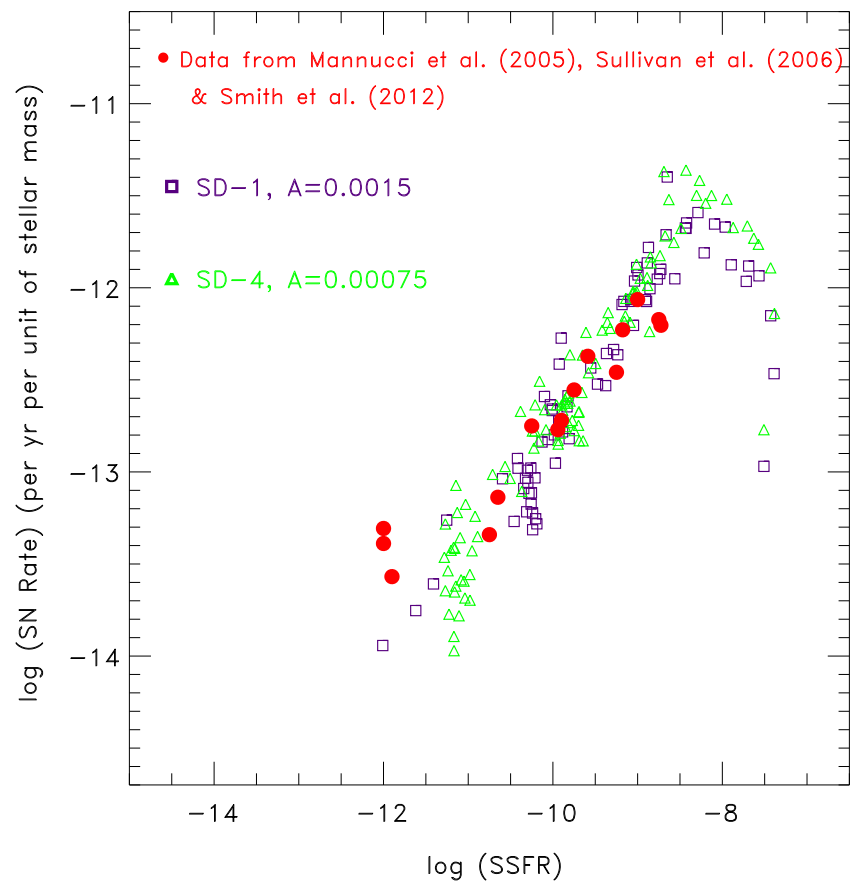

Figure 5. Specific star formation rate (sSFR) as a function of the SN Ia rate per unit of galaxy mass (SSNIaR) for the SD-1 and SD-4 models (runs with $A=0.0015$ and $A=0.00075)$. The red circles represent combined data from Mannucci et al. (2005), Sullivan et al. (2006), and Smith et al. (2012). Notice that these models also fit the observed $[\mathrm{O} / \mathrm{Fe}]$ ratios coming from the Galactic bulge and the present-day SN Ia rates. The zero point has been renormalized by an ad hoc factor. See Section 5 for further details.

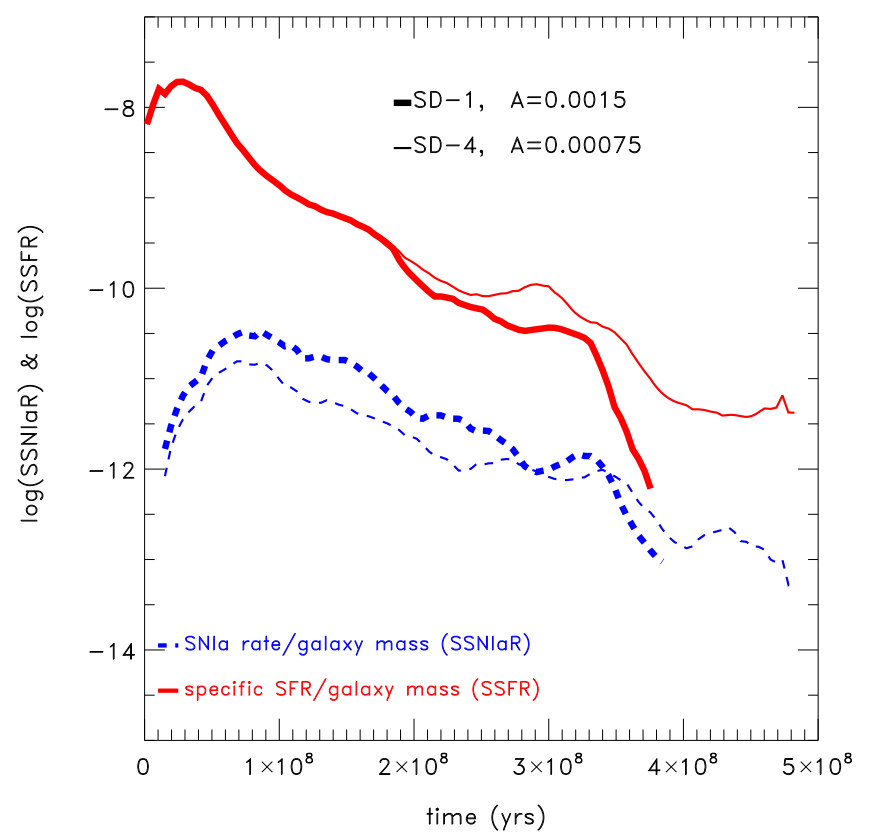

Figure 6. The $\log (\mathrm{sSFR})$ (solid line) and $\log (\mathrm{SSNIaR})$ (dashed line), evolving with time for our best models within the SD scenario (SD-1 and SD-4).

\section{DISCUSSION AND CONCLUSIONS}

We present results from simulated galaxies performed with a version of Tree-PM SPH-GADGET-3 (Springel 2005; Scannapieco et al. 2006), to study the impact of the SN Ia feedback by using different DTDs. In this paper, we explore the SD 
model of MR01 in detail. We choose the SD scenario as a reference to discuss the implementation, the main observables, and correlations that we aim to reproduce. The implementation of the SD scenario involves the calibration of a free parameter $A$, which represents the fraction of binary systems in one stellar generation giving rise to $\mathrm{SN}$ Ia events. Chemical evolution models generally fix $A$ according to the SFR and the presentday SN Ia rates of the galaxy under study. However, in cosmological simulations $A$ acts at a particle basis. Thus, it is assumed to be the same for all single stellar populations, while the final SN Ia rates or any relation between the SN Ia rates and the galaxy properties - or the chemical abundance patternsshould come out as a prediction of the simulations. Therefore, it is necessary to explore a range of suitable $A$ values and their impact on the properties of galaxies, by using simple initial conditions. In a forthcoming paper, we explore other progenitor scenarios. The final goal is to run cosmological simulations and analyze the chemical properties of galaxies of different masses and assembly histories. Adequately reproducing the SN Ia feedback, as well as their effect on the chemical enrichment, is very important, as new Galactic surveys will start yielding high-precision measurements of chemical abundances.

Our main results can be summarized as follows:

1. We find that the SFR responds linearly to the number of $\mathrm{SNe}$ Ia in the SD scenario. The SFR is found to decline faster with increasing values of $A$. But the differences are small, and so the final stellar mass formed is very similar. There is a substantial difference between the original model of Scannapieco et al. (2006) and the SD of Matteucci \& Recchi (2001) implemented and analyzed here. This is related to the adopted lifetimes for the secondary masses. In the original model, the $\mathrm{SN}$ Ia production was shifted to later times, compared to the SD scenario, beginning at $\sim 1 \times 10^{8} \mathrm{yr}$. This excludes the socalled prompt SNe Ia, which are indeed observed. For a similar $A$, the original model shows a more extended SFR. However, both of them predict similar final fractions of new stellar mass to total baryonic mass.

2. The SFR in the simulations is dominated by a strong starburst consuming the cold gas within $\sim 1 \mathrm{Gyr}$ and forming stars that end up concentrated within the bulge. Hence, the SN Ia rates are comparable to observed ones for spheroidal galaxies. This comparison with observed SN Ia rates of $\mathrm{Li}$ et al. (2011) shows that the best fits are obtained for SD-1 and SD-4 models, adopting $A \sim 0.0015$ and $A \sim 0.00075$, respectively.

3. The $[\mathrm{O} / \mathrm{Fe}]$ ratios predicted for the stars in the bulge of the simulated galaxies are compared with data from the Galactic bulge (although this comparison is just indicative). We find that the best agreement with observations is again provided by the SD-1 and SD-4 models. These models predict a long plateau for the $[\mathrm{O} / \mathrm{Fe}]$ ratio and a knee occurring at high $[\mathrm{Fe} / \mathrm{H}]$, as observations suggest. We note that this is the first time that such an excellent agreement is found in galaxy simulations.

4. At variance with previous claims, we find that the SD scenario (SD-1 and SD-4 models) reproduces the observed correlation between the SSNIaR and the SSFR, found by Sullivan et al. (2006), if we estimate these quantities at different evolutionary times. This correlation comes out naturally from the simulations. Moreover, two features in the correlations are shown that cannot be confronted with current observations but could be interesting to explore in the future:

For high sSFR, as the SSNIaR starts to appear, the SSNIaR-sSFR anticorrelates before it turns into the observed correlation. This is caused by the large initial number of SNe Ia in the assumed DTD. The turnover occurs when the maximum in the DTD is reached; hence, it could be interesting to explore if this turnover could be confirmed with observations coming from galaxies dominated by a very recent starburst.

For very low sSFR, there is still a residual SSNIaR because the majority of these events are delayed in time, and thus the correlation disappears. The sSFR at which this occurs could be related to the shape of the DTD.

In a forthcoming paper, we will discuss other DTDs implemented in the SPH simulations: the DD scenario by Greggio (2005) and empirically motivated DTDs such as the bimodal scenario by Mannucci et al. (2006) and the power laws reported by Maoz et al. (2012) and Pritchet et al. (2008). We will explore the predicted $\mathrm{SN}$ Ia rates and chemical abundances, as well as the global correlations that can contribute to the understanding of SN Ia progenitors.

N.J. is very grateful for fruitful discussions and comments from Marcelo Miller Bertolami, Ilaria Bonaparte, Emanuele Spitoni, Laura Greggio, Jordi Isern, Nikos Pranzos, Robert Yates, Manuela Zoccali, Thomas Bensby, Enrique Gaztaniaga, Emanuel Sillero, and Kai Hoffmann. Many thanks to Kate Rowlands and Jack A. Kennedy for their comments and corrections to the manuscript. We thank the anonymous referee for a very constructive report that helped to clarify the paper.

We thank Mark Sullivan and Mathew Smith for kindly providing the data shown in this paper.

N.J. acknowledges support from CONICET-Argentina; the European Research Council Starting Grant (SEDmorph; P.I. V. Wild); and the European Commission's Framework Programme 7, through the Marie Curie International Research Staff Exchange Scheme LACEGAL (PIRSES-GA-2010269264), and warmly thanks the Observatory of Trieste and the Institute of Space Studies of Catalunya, where most of this work was done.

This work was partially supported by PIP 2009/0305 (CONICET-Argentina) and PICT Raices 2011/959 (FoncytArgentina). Simulations where run in Fenix Cluster of the Institute of Astronomy and Space Physics (IAFE-Argentina) and Hal Cluster of the Universidad Nacional of Cordoba (Argentina). Also, this work was supported by PIP 2009/0305 (CONICET-Argentina), PICT Raices 2011/959 (FoncytArgentina), "Proyecto Interno" of the Universidad Andres Bello (Chile), and Fondecyt Regular 2015 1150334. F.M. acknowledges financial support from PRINMIUR2010-2011, project: "Chemical and dynamical evolution of the Milky Way and Local Group Galaxies," prot.N. 2010LY5N2T. This research made use of the NASA's Astrophysical Data Systems and the arXiv.org e-print service from Cornell University.

\section{REFERENCES}

Anders, F., Chiappini, C., Santiago, B. X., et al. 2013, arXiv:1311.4549 Arrigoni, M., Trager, S. C., Somerville, R. S., \& Gibson, B. K. 2010, MNRAS, 402, 173 
Artale, M. C., Tissera, P. B., \& Pellizza, L. J. 2015, arXiv:1502.00017 Aumer, M., White, S. D. M., Naab, T., \& Scannapieco, C. 2013, MNRAS, 434, 3142

Bensby, T., Yee, J. C., Feltzing, S., et al. 2013, A\&A, 549, A147

Bianchi, S., \& Schneider, R. 2007, MNRAS, 378, 973

Bonaparte, I., Matteucci, F., Recchi, S., et al. 2013, MNRAS, 435, 2460

Bravo, E., Isern, J., \& Canal, R. 1993, A\&A, 270, 288

Brocato, E., Matteuci, F., Mazzitelli, I., \& Tornambe, A. 1990, ApJ, 349, 458

Calura, F., Gibson, B. K., Michel-Dansac, L., et al. 2012, MNRAS, 427, 1401

Calura, F., \& Menci, N. 2009, MNRAS, 400, 1347

Cappellaro, E., Mazzali, P. A., Benetti, S., et al. 1997, A\&A, 328, 203

Carraro, G., Lia, C., \& Chiosi, C. 1998, MNRAS, 297, 1021

Chiappini, C., Matteucci, F., \& Romano, D. 2001, ApJ, 554, 1044

Croom, S. M., Lawrence, J. S., Bland-Hawthorn, J., et al. 2012, MNRAS, 421, 872

De Lucia, G., Tornatore, L., Frenk, C. S., et al. 2014, MNRAS, 445, 970

Few, C. G., Courty, S., Gibson, B. K., Michel-Dansac, L., \& Calura, F. 2014, MNRAS, 444, 3845

Fioc, M., \& Rocca-Volmerange, B. 1997, A\&A, 326, 950

François, P., Matteucci, F., Cayrel, R., et al. 2004, A\&A, 421, 613

Gargiulo, I. D., Cora, S. A., Padilla, N. D., et al. 2015, MNRAS, 446, 3820

Geier, S., Marsh, T. R., Wang, B., et al. 2013, A\&A, 554, A54

Greggio, L. 1996, in Proc. of the 11th IAP Astrophysics Meeting, The Interplay between Massive Star Formation, the ISM and Galaxy Evolution, ed.

D. Kunth et al. (Paris: AIP), 89

Greggio, L. 2005, A\&A, 441, 1055

Greggio, L. 2010, MNRAS, 406, 22

Greggio, L., \& Renzini, A. 1983, A\&A, 118, 217

Hachisu, I., Kato, M., \& Nomoto, K. 1996, ApJL, 470, L97

Hachisu, I., Kato, M., \& Nomoto, K. 1999, ApJ, 522, 487

Han, Z., \& Podsiadlowski, P. 2004, MNRAS, 350, 1301

Hoyle, F., \& Fowler, W. A. 1960, ApJ, 132, 565

Iben, I., Jr., \& Tutukov, A. V. 1984, ApJS, 54, 335

Iwamoto, K., Brachwitz, F., Nomoto, K., et al. 1999, ApJS, 125, 439

Jiménez, N., Cora, S. A., Bassino, L. P., Tecce, T. E., \& Smith Castelli, A. V. 2011, MNRAS, 417, 785

Kennicutt, R. C., Jr. 1998, ApJ, 498, 541

Kobayashi, C. 2004, MNRAS, 347, 740

Kobayashi, C., Tsujimoto, T., Nomoto, K., Hachisu, I., \& Kato, M. 1998, ApJL, 503, L155

Kroupa, P. 2001, MNRAS, 322, 231

Lecureur, A., Hill, V., Zoccali, M., et al. 2007, A\&A, 465, 799

Li, W., Chornock, R., Leaman, J., et al. 2011, MNRAS, 412, 1473

Longhetti, M., \& Saracco, P. 2009, MNRAS, 394, 774

Lorén-Aguilar, P., Isern, J., \& García-Berro, E. 2010, MNRAS, 406, 2749

Maiolino, R., Schneider, R., Oliva, E., et al. 2004, Natur, 431, 533

Mannucci, F., Della Valle, M., \& Panagia, N. 2006, MNRAS, 370, 773

Mannucci, F., Della Valle, M., Panagia, N., et al. 2005, A\&A, 433, 807

Maoz, D., Mannucci, F., \& Brandt, T. D. 2012, MNRAS, 426, 3282

Maoz, D., Mannucci, F., \& Nelemans, G. 2013, arXiv:1312.0628

Matteucci, F. 1994, A\&A, 288, 57

Matteucci, F., \& Brocato, E. 1990, ApJ, 365, 539

Matteucci, F., \& Greggio, L. 1986, A\&A, 154, 279

Matteucci, F., Panagia, N., Pipino, A., et al. 2006, MNRAS, 372, 265

Matteucci, F., \& Recchi, S. 2001, ApJ, 558, 351

Matteucci, F., Spitoni, E., Recchi, S., \& Valiante, R. 2009, A\&A, 501, 531

McWilliam, A., Matteucci, F., Ballero, S., et al. 2008, AJ, 136, 367

Mosconi, M. B., Tissera, P. B., Lambas, D. G., \& Cora, S. A. 2001, MNRAS, 325,34

Nagashima, M., Lacey, C. G., Baugh, C. M., Frenk, C. S., \& Cole, S. 2005, MNRAS, 358, 1247
Nakasato, N., \& Nomoto, K. 2003, ApJ, 588, 842

Navarro, J. F., Frenk, C. S., \& White, S. D. M. 1997, ApJ, 490, 493

Padovani, P., \& Matteucci, F. 1993, ApJ, 416, 26

Pan, T., Kasen, D., \& Loeb, A. 2012, MNRAS, 422, 2701

Perez, J., Michel-Dansac, L., \& Tissera, P. B. 2011, MNRAS, 417, 580

Perez, J., Valenzuela, O., Tissera, P. B., \& Michel-Dansac, L. 2013, MNRAS, 436, 259

Perlmutter, S., Turner, M. S., \& White, M. 1999, PhRvL, 83, 670

Pietrinferni, A., Cassisi, S., Salaris, M., \& Castelli, F. 2006, ApJ, 642, 797

Pipino, A., \& Matteucci, F. 2004, MNRAS, 347, 968

Pritchet, C. J., Howell, D. A., \& Sullivan, M. 2008, ApJL, 683, L25

Raiteri, C. M., Villata, M., \& Navarro, J. F. 1996, A\&A, 315, 105

Rest, A., Scolnic, D., Foley, R. J., et al. 2013, arXiv:1310.3828

Rojas-Arriagada, A., Recio-Blanco, A., Hill, V., et al. 2014, arXiv:1408.4558

Salpeter, E. E. 1955, ApJ, 121, 161

Sanchez, S. F., Rosales-Ortega, F. F., Iglesias-Paramo, J., et al. 2013, arXiv: 1311.7052

Scannapieco, C., Tissera, P. B., White, S. D. M., \& Springel, V. 2005, MNRAS, 364, 552

Scannapieco, C., Tissera, P. B., White, S. D. M., \& Springel, V. 2006, MNRAS, 371, 1125

Scannapieco, C., Tissera, P. B., White, S. D. M., \& Springel, V. 2008 MNRAS, 389, 1137

Scannapieco, C., White, S. D. M., Springel, V., \& Tissera, P. B. 2009 , MNRAS, 396, 696

Schmidt, M. 1959, ApJ, 129, 243

Shen, K. J., Guillochon, J., \& Foley, R. J. 2013, ApJL, 770, L35

Smith, M., Nichol, R. C., Dilday, B., et al. 2012, ApJ, 755, 61

Soker, N., García-Berro, E., \& Althaus, L. G. 2013, MNRAS, arXiv: 1309.0368

Springel, V. 2005, MNRAS, 364, 1105

Sullivan, M., Le Borgne, D., Pritchet, C. J., Hodsman, A., \& Neill, J. D. 2006, ApJ, 648, 868

Sutherland, R. S., \& Dopita, M. A. 1993, ApJS, 88, 253

Tinsley, B. M. 1979, ApJ, 229, 1046

Tissera, P., Beers, T., Carollo, D., \& Scannapieco, C. 2013a, arXiv:1309.3609

Tissera, P. B., Beers, T. C., Carollo, D., \& Scannapieco, C. 2014, MNRAS, 439, 3128

Tissera, P. B., Scannapieco, C., Beers, T. C., \& Carollo, D. 2013b, MNRAS, 432,3391

Tissera, P. B., White, S. D. M., \& Scannapieco, C. 2012, MNRAS, 420, 255

Tornambe, A., \& Matteucci, F. 1987, ApJL, 318, L25

Tornatore, L., Borgani, S., Matteucci, F., Recchi, S., \& Tozzi, P. 2004, MNRAS, 349, L19

Totani, T., Morokuma, T., Oda, T., Doi, M., \& Yasuda, N. 2008, PASJ, 60, 1327

Tsebrenko, D., \& Soker, N. 2013, MNRAS, 435, 320

Tutukov, A. V., \& Yungelson, L. R. 1980, SvAL, 6, 271

Valiante, R., Matteucci, F., Recchi, S., \& Calura, F. 2009, NewA, 14, 638

van den Bergh, S. 1991, ApJ, 369, 1

van den Heuvel, E. P. J., Bhattacharya, D., Nomoto, K., \& Rappaport, S. A. 1992, A\&A, 262, 97

Whelan, J., \& Iben, I., Jr. 1973, ApJ, 186, 1007

White, S. D. M., \& Frenk, C. S. 1991, ApJ, 379, 52

Wiersma, R. P. C., Schaye, J., Theuns, T., Dalla Vecchia, C., \& Tornatore, L. 2009, MNRAS, 399, 574

Woosley, S. E., \& Weaver, T. A. 1995, ApJS, 101, 181

Yates, R. M., Henriques, B., Thomas, P. A., et al. 2013, MNRAS, 435,3500

Zoccali, M., Gonzalez, O. A., Vasquez, S., et al. 2014, arXiv:1401.4878 